\title{
ARM Based Smart Living System using Brain Computer Interface
}

\author{
K. Arun Kumar ${ }^{1}$, R. Satya Prakash ${ }^{2}$, and M. Vinod Kumar Reddy ${ }^{3}$ \\ ${ }^{1}$ Asst. Professor, CVR College of Engineering/ ECE Department, Hyderabad, India \\ Email: arun.katkoori@gmail.com \\ ${ }^{2}$ Asst. Professor, CVR College of Engineering/ CSE Department, Hyderabad, India \\ Email: prakashscits@gmail.com \\ ${ }^{3}$ Asst. Professor, CVR College of Engineering/ECE Department, Hyderabad, India \\ Email: vinodreddy.488@gmail.com
}

\begin{abstract}
As of late, numerous ecological control systems have been proposed to improve human personal satisfaction. In any case, little research has concentrated on environmental control directly using the human physiological state. The primary point of this paper is to control the physical gadgets like home machines utilizing EEG signals and to assist the physically disabled persons with controlling the home apparatuses, so they become autonomous in their everyday life. This methodology offers an elective correspondence and the control framework and is referred as an artificial system that evades the human body's typical enough pathways, which are neuromuscular yield channels. In this paper, a non-obtrusive Brain Computer Interface is utilized. The brainwave sensor is utilized to detect the brain signals and eye blink. As per the eye blink values and the brain attention values, the gadgets will be chosen and through transfers the turning ON and OFF the home apparatus are done as needs be. The experimental results show how to operate the modules like fan/bulb by blinking the eyes with help of ARM7 processor. It is observed that the mean response time of the system for bulb was found to be $15.6 \mathrm{sec}$ with accuracy of $84.6 \%$ for $O N$ state, and 21 sec with accuracy of $72.4 \%$ for OFF state.
\end{abstract}

Index Terms: Brain wave sensor, Brain computer Interface, Electroencephalogram (EEG), ARM processor.

\section{I.INTRODUCTION}

Recently, with the development in sensor innovation and data technology, numerous examinations are attempting to create business products to acquire the comfort to individuals their standard life. In this manner, the fast development of research on smart houses is proposed and created as a standard to give different sorts of environmental control systems. RFID, outside sensor modules, and voice recognition are used as control signals in some of the environmental control systems [1]. For automatic detection of person's motion, the RFID tags or external sensors are placed in different regions in advance. By combining with universal plug and play home systems, a person can send a request from his cell phones, a wearable machine, or outside sensors to the home server either motion, GUI, or voice. Brain Computer Interface is an alternative technique to apply the physiological signals as the stimulus of ecological control system in smart home [2][3].

In any case, the greater part of current BCI based system requires the person's dynamic mental order to control outside devices [4][5] [6]. Subsequently, these systems can't control peripherals consequently and adaptively as indicated by the client's present intellectual state. Besides, most of the flow BCI-based environmental systems are inconvenient because of their complexity and expensive.

Therefore, the goal of this paper is to propose a low cost and flexible brain computer interface-based system to control devices based on the user's phycological state (languor or readiness). In this method, an embedded processor like ARM processor and signal acquisition module like brain wave sensor are proposed. Not quite the same as other BCI frameworks, which are generally massive and need to transmit EEG signals to a backend PC to process the EEG signal, our proposed method is having the advantages of low power, small size and are suitable for practical applications.

\section{Proposed Model}

\section{A. EXISTING METHOD}

In a normal system, to control electronic home appliances a manual method of operating is required and there is no means of data transfer. The data transfer is done through cables and individual must depend upon others to operate the devices. And, there is no muscle contraction in existing systems where as BCIs use brain signals to gather information on user intentions[7]. To that effect, BCIs rely on recording state that measures brain activity and translates the information into tractable electrical signals and requires direct personal computer interaction.

In the proposed system, the basic method adapted is Brain wave analysis, where the signals generated from the brain are directly analyzed. A thought generated by the brain can make certain muscular contractions, by this an automated robot could be controlled by using human thoughts[8]. The proposed system provides self-controlled and operating facility. A most of newly developed devices are features with Bluetooth which is easy to access and transfer files in the proposed system Bluetooth communication plays a major role in transferring the data for analysis[9]. The architecture of Brain Computer Interface is shown in Figure 1. It contains three stages- i) signal acquisition and preprocessing, ii) Signal processing, iii) commands/ control. 


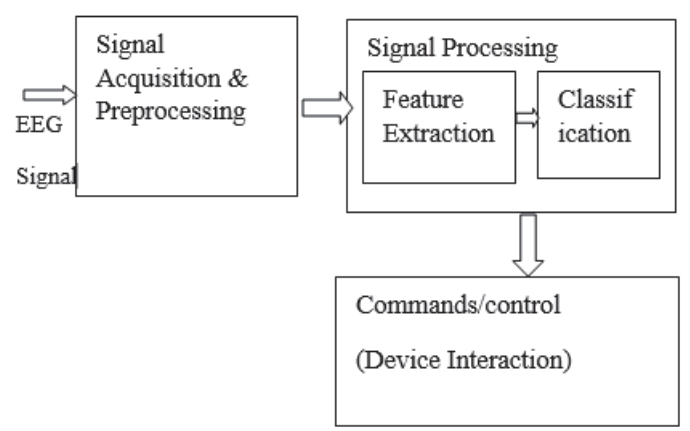

Figure 1. BCI Structure

\section{B. EEG-ELECTROENCEPHALOGRAM}

The EEG is a chronical of the electrical movement of the brain from the scalp. The principal recordings were made by Hans Berger in 1929 although comparative investigations had been completed in creatures as ahead of schedule as 1870 .

The waveforms recorded are thought to mirror the movement of the outside of the brain, the cortex. This movement is impacted by the electrical action from the mind structures underneath the cortex. EEG follows are appeared in figure 2. Each electrode is marked with a letter followed by a number, the letter refers to the zone of cerebrum basic the electrode and the number is even or odd. Odd number indicates left half of the head and even indicates the correct side of the head.

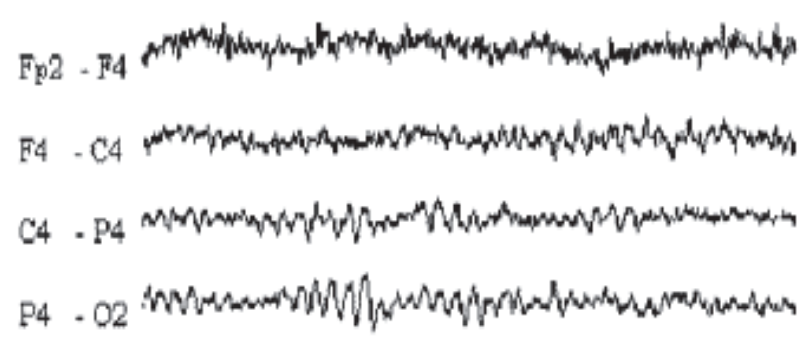

Figure 2. EEG traces

The nerve cells in the mind produce a signal that is called activity over possibilities. These active possibilities move to start with one cell then onto the next over a hole called the neural connection. Unique synthetic compounds called synapses help the signal to move over the gap. There are two kinds of synapses; one will help the active potential to move to the following cell, the other will stop it moving to another nerve cell. The brain ordinarily strives to keep an equivalent measure of every one of these synapses in the mind. EEG action is very little, estimated in microvolts with the primary frequencies of interest up to around $30 \mathrm{~Hz}$.
To examine the EEG signal, a 'Thinkgear' brainwave sensor is utilized. Brain-Computer-Interface is an immediate correspondence pathway between human brain and the external component. As per the human contemplations i.e., the brain consideration esteems the physical components are worked [10]. The signal acquisition unit receives the brain wave raw data and processes the signal utilizing MATLAB. Along with MATLAB, Visual Basic software is used because, a large amount of data is receiving from the brain sensor and sending these data to the MATLAB in signal processing unit [11][12]. Meanwhile the attention and eye blink values are sent to the hardware side. So, the data transmission rate in Visual Basic is relatively more prominent than that of the MATLAB. The following figure 3 shows the block diagram of BCI.

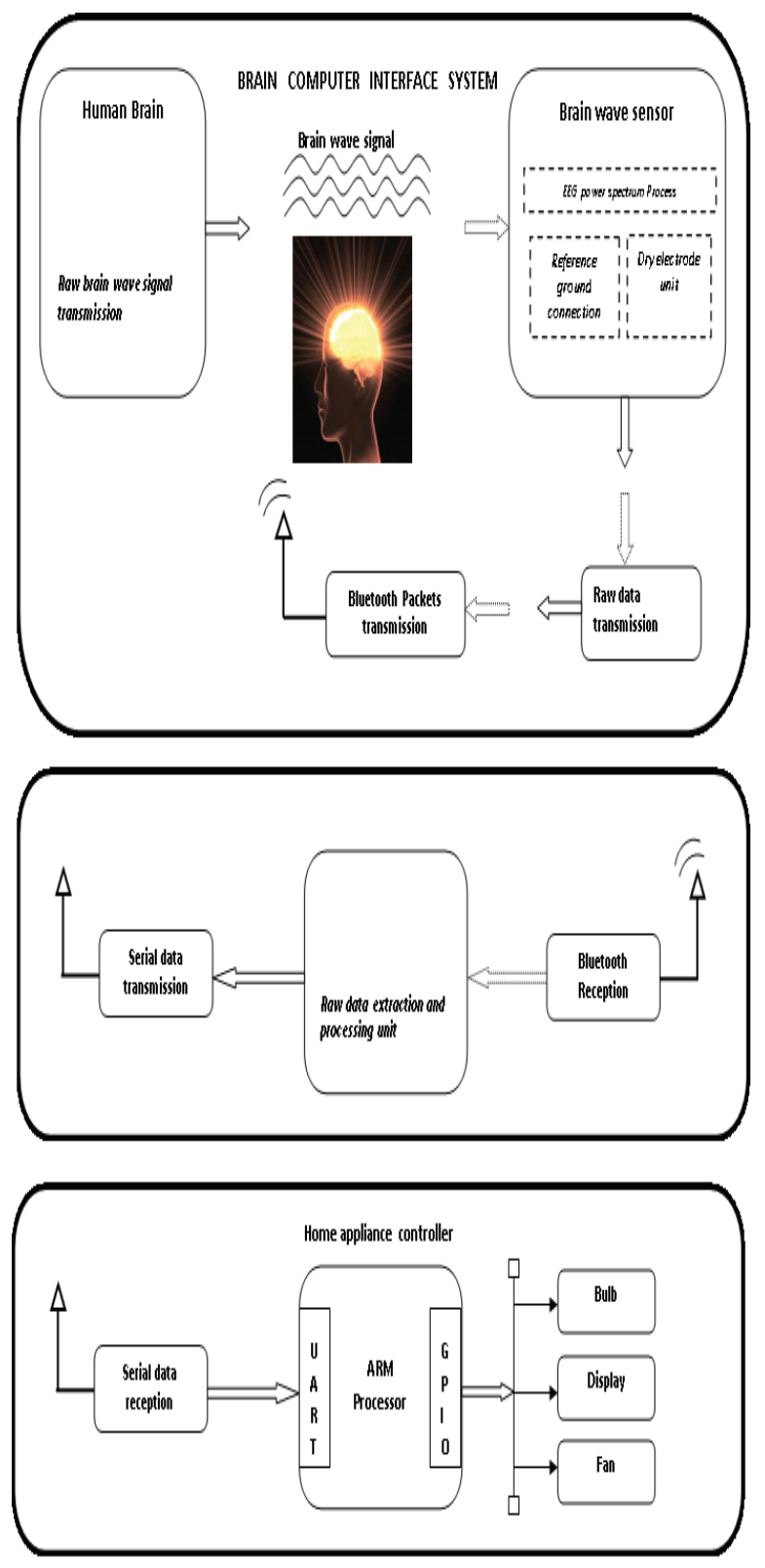

Figure 3. Proposed BCI system Block diagram 
The brain wave data is sent to the controller unit. This data is sent serially using UART. Then the instructions will be sent to the home appliances to control the devices like bulb, fan, display, etc. as shown figure 3. In the controller unit, ARM controller receives serial data and send commands to the physical devices through GPIO bus.

\section{ARM PROCESSOR}

The abbreviation of ARM is Advanced RISC Machine, is widely used in the field of embedded system. The following are the features of ARM3 module-

-It's Operating clock frequency varies from $156 \mathrm{MHz}$ to $320 \mathrm{MHz}$.

- Contains two 16-bit MAC (multiply-and-accumulate) units to execute 1200 lines multiplication and addition operations.

- It has four independent DMA (Direct Memory Access) mechanisms. The processing time was reduced.

-It contains Serial peripheral interface Flash is used (to reduce the module size).

- It also contains wireless transmission unit via universal asynchronous receiver/ transmitter (UART) interface.

- This module can be operated with a $3.7 \mathrm{~V}$ DC power supply, and it can continuously operate for more than $45 \mathrm{~h}$ operations with a $1100 \mathrm{mAh}$ Li-ion battery.

Cognitive state detection algorithm was implemented in this embedded module. Then got EEG information is processed, analyzed and displayed by this module [13]. At this point when the difference is subjective condition of the client is identified, the corresponding instruction will be sent either by RF module or by Ethernet through Universal plug and play protocol. A RS232 to Ethernet adapter module is required to do this.

\section{BRAIN SENSE-BRAIN WAVE SENSOR}

Brain wave is a Brain-Computer Interface (BCI) device transforms person's brainwaves into activities, opening new worlds of intuitiveness [14]. By using proprietary Attention and Meditation eSense algorithms, the brain wave sensor reports the wearer's mental state. The head set of brain wave sensor is shown in figure 4. Implanted inside the TGAM, is the TGAT chip, a ground-breaking, completely coordinated single chip EEG sensor. The chip comes modified with Neurosky eSense, Analog-to-Digital Converter, enhancement off head recognition, and noise filtering for EMG and 50/60 Hertz AC powerline impedance. The TGAM is evaluated to control mass reception in toys, cell phones, instructive apparatus and different items.

To power ON the Brainwave sensor, plug the battery terminal to the terminals. To turn the Brainwave sensor OFF, remove the terminals plugged to the battery. [15] While the Brainwave is powered on, the LED light on the side of the headset will be turned on.

Brainsense is more than an average headset. It can use person's brainwaves for exciting new applications. In order to take full advantage of these functions and features of the Brainsense, the Brainsense must be properly worn.

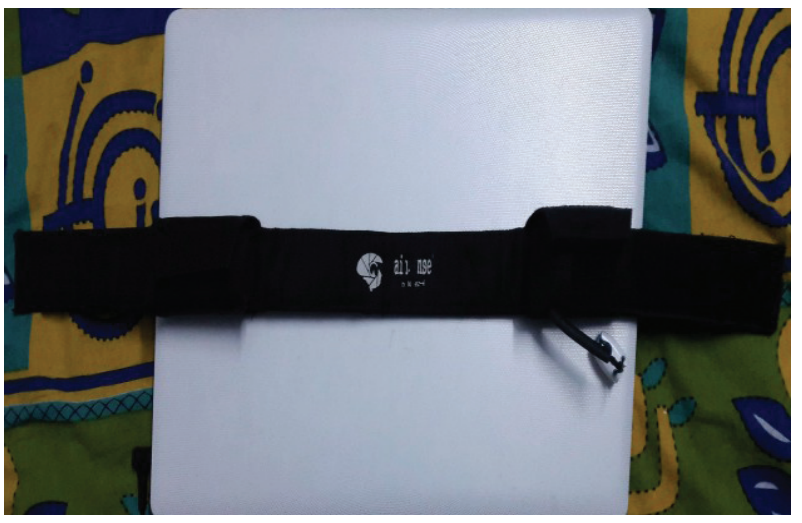

Figure 4. Brain wave sensor headset

\section{Features and functions of Brain wave sensor:}

It allows the ear band to rest behind the left ear, and afterward clip the ear clip onto individual's ear cartilage.

Make sure the two metal contacts within the two sides of the ear clip make skin contact with ear. Move any hair or obstructions such as jewelry out of the way. Read just the ear clip as necessary to make proper contact with the skin of ear. The figure 5 shows the brain sense earlobe. It may need to squeeze the ear clip against human's ear for a few moments.

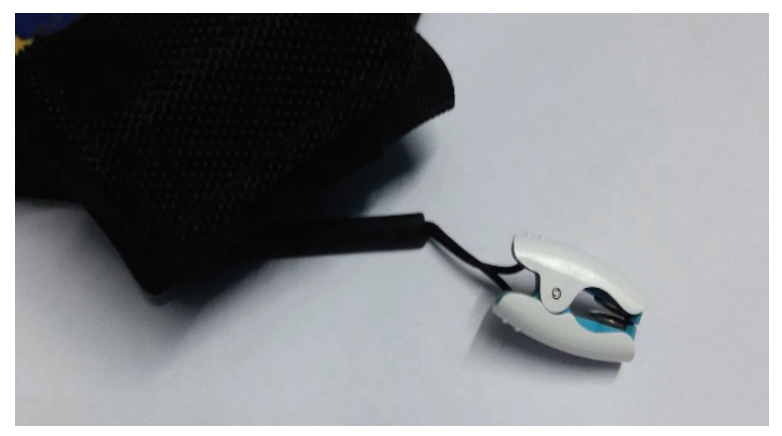

Figure 5. Brainsense Earlobe

Adjust the forehead Sensor Arm of the headset so that the Sensor Tip contacts the skin of forehead. Sensor Tip must maintain steady skin contact in order to properly measure your brainwaves.

Sensor Tip should be comfortable yet stay normally in position. Keep hair away from the sensor - the sensor must always be able to directly contact the skin. Make up, dead skin, or debris can interfere with the connection. Scratch or wipe the obstruction away if the person has trouble obtaining a clean signal.

The Brainsense should look when properly worn. During usage, if person is not receiving a signal, repeat the steps above to make minor adjustments to ensure the sensor and contacts have proper skin contact. Figure 6 shows the brain sense forehead sensor tip contact. 


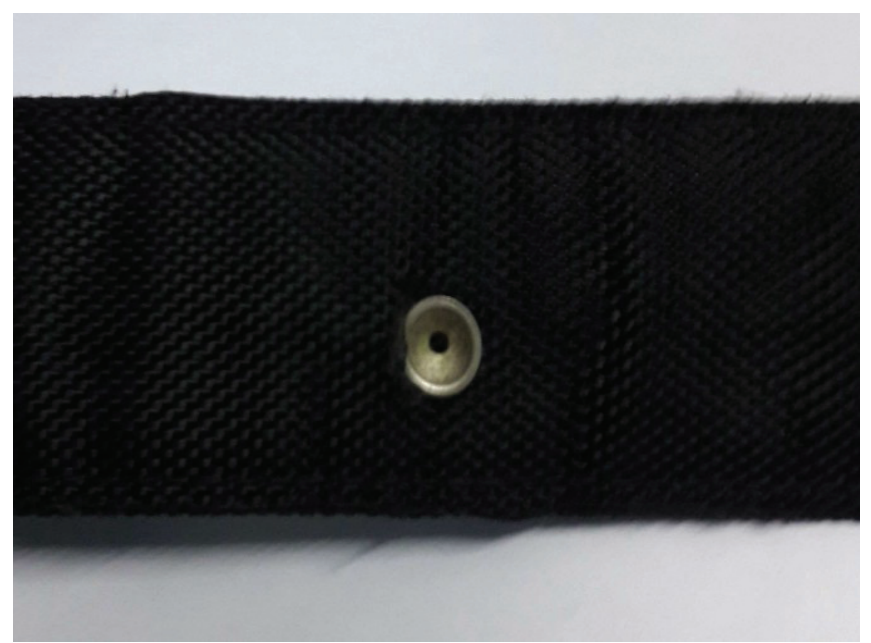

Figure 6. Brain sense forehead sensor tip contact

\section{E.FLOWCHART:}

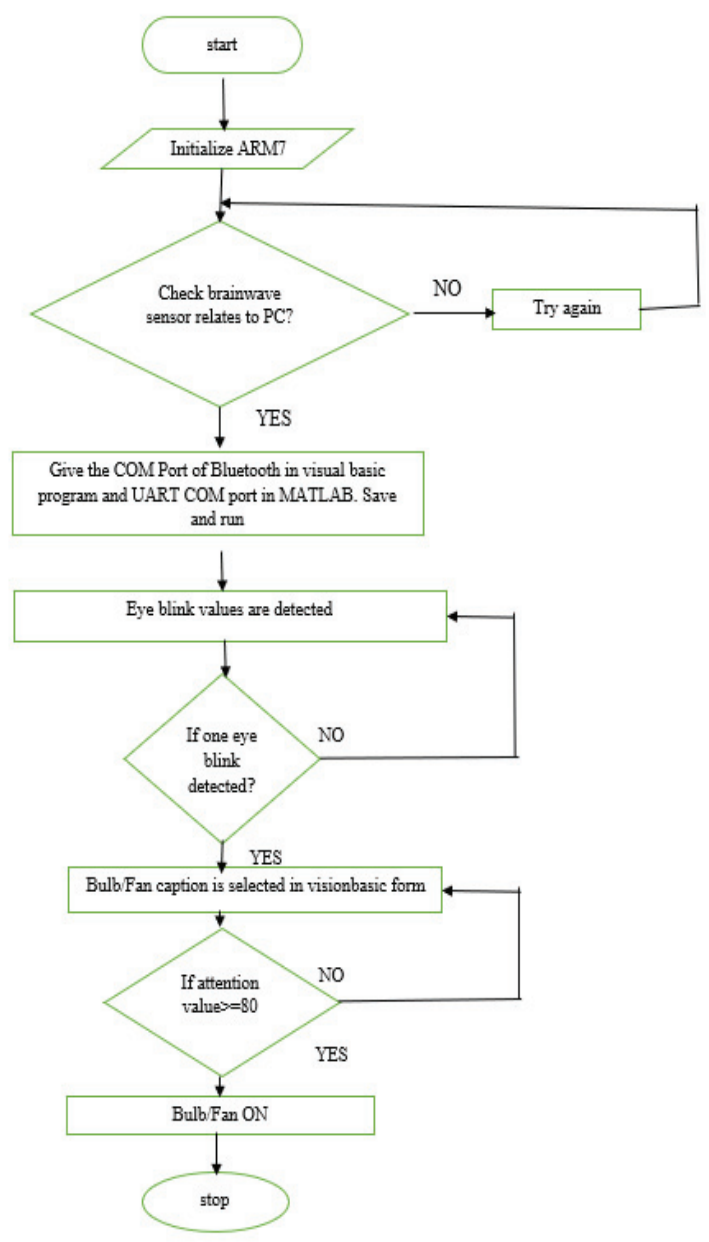

Figure 7. Flowchart of proposed method

\section{F. HARDWARE CONNECTIONS}

- Establish a connection between the board to the power supply.

- Connect the adapter to the DC voltage and plug it to the LPC2148 board and switch on the power supply.

- Switch on the thinkgear Brain wave sensor by plugging the battery, check whether the LED is turned on and blinking.

- Check whether the dry electrode is properly in contact with the forehead and the ear plug is properly attached to the ear lobe with proper contact.

- Check whether the COM port and Bluetooth is properly paired with the PC and sensor. These are shown in figure 8.

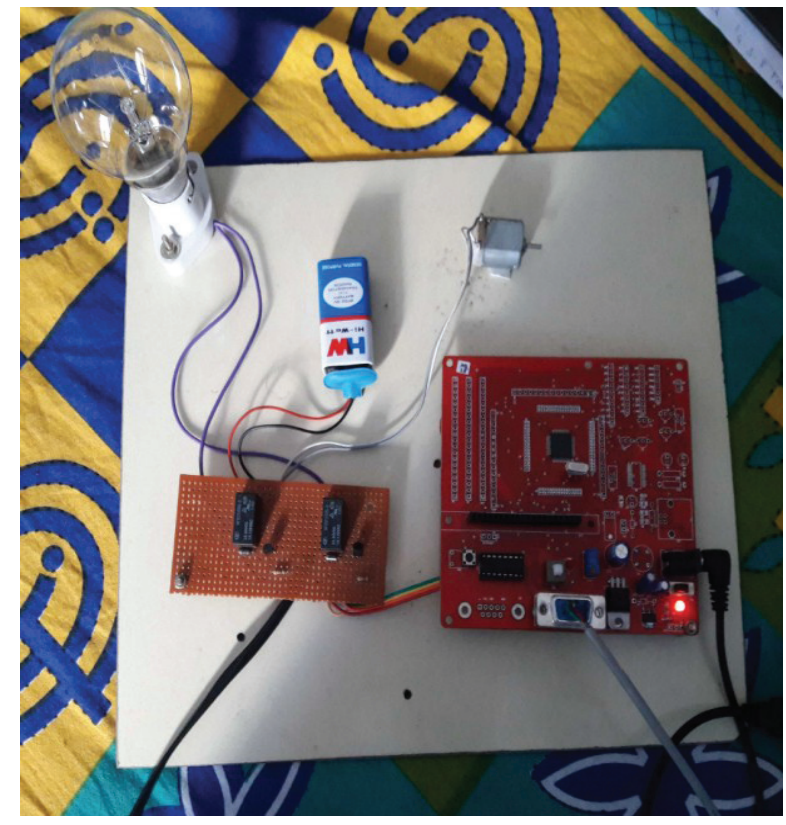

Figure 8. Hardware connections

\section{G. RESULTS}

- In order to make the application run,Open the Matlab M-File.

- Click the run button in the editor toolbox.

- After clicking the run button, a new command window opens, The thinkgear is loaded and the attention and meditation values are plotted

- These signals are plotted and analyzed by the brainwave sensor.

- The muscle contraction occurs because of blinking of the eyes and the bulb turns on and off accordingly.

- In the figure 9, the red line in the graph represents the attention value and the black color dots represent the number of times the blink is plotted. The form of Visual Basic is displayed when the 
value of eye blink detected is 80 . A device is selected depending on number of eye blinks (one or two eye blinks) and that device is tuned $\mathrm{ON}$ or OFF depending on the attention value.

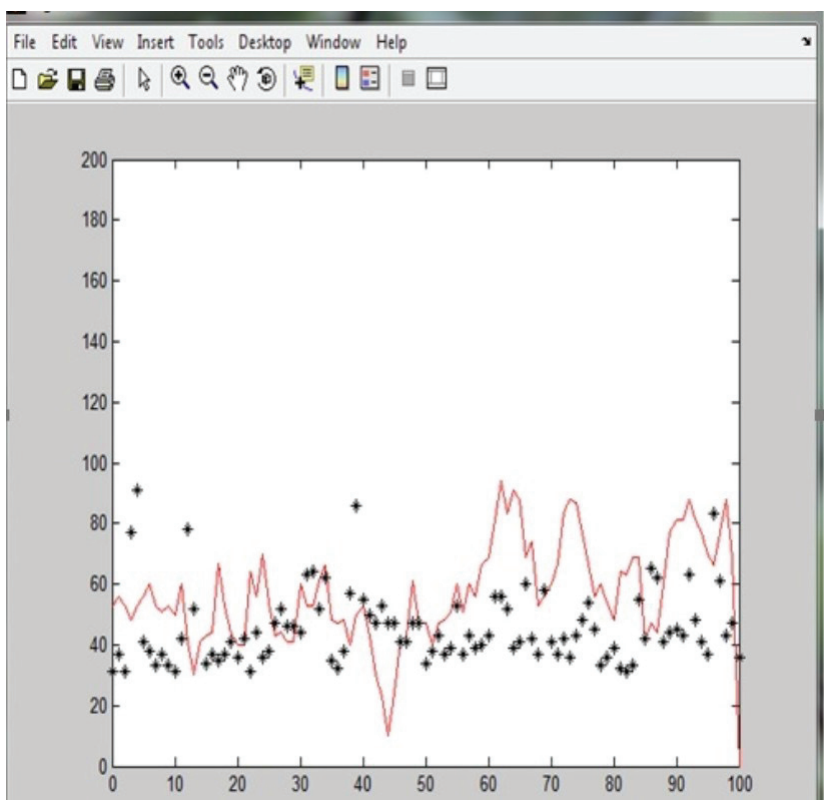

File Edit View Insert Tools Desktop Window Help
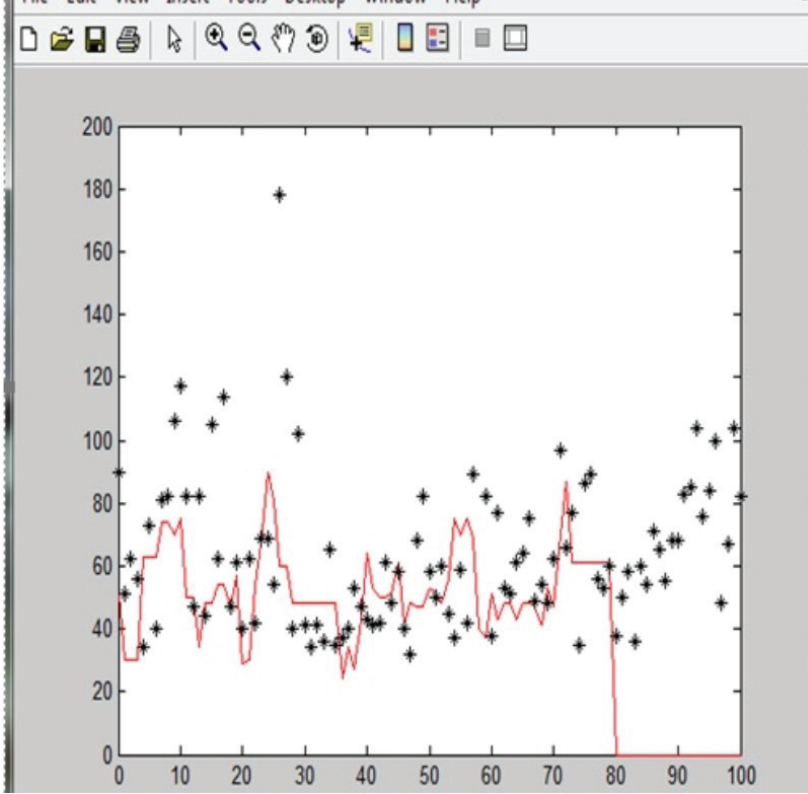

Figure 9. Graphical plotting for different number of trials

Vision Basic is a Field Device Tool frame application with a modern \& uncomplicated GUI. It gives powerful essential usefulness to setup, diagnostics and support of intelligent field gadgets.

Different patterns of neural interaction lead to different waveforms. These waves are having different amplitudes and frequencies. This neural cooperation is finished with different neurons. Each collaboration between neurons makes a tiny elctrical release.
After connecting all the components switch ON the power supply.In a few seconds thinkgear brainwave sensor establishes a connection to the personal computer via Bluetooth.the signal generated is analyzed by using the MATLAB GUI platform. Then the instructions will be sent to the home section to operate the modules like bulb and fan, etc. The ON-OFF condition of elctrical gadjets is based on changing the muscle movement with eye blinking.

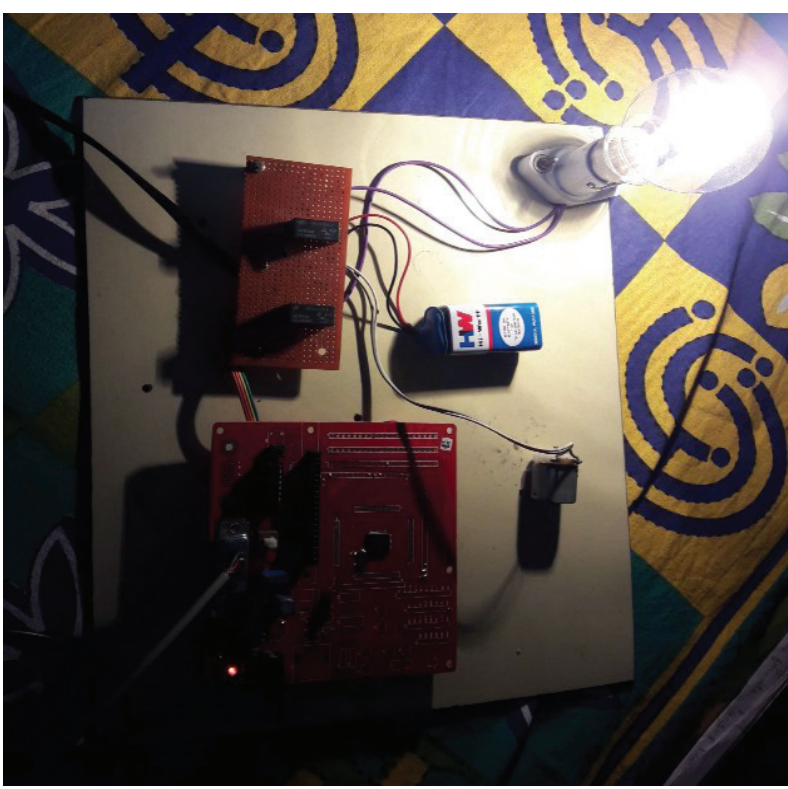

Figure 10. output of the module

TABLE I.

MEAN Response Time For BUlb for Two StATES

\begin{tabular}{|l|l|l|}
\hline Number of trials & ON state(sec) & $\begin{array}{l}\text { OFF } \\
\text { state(sec) }\end{array}$ \\
\hline Trial 1 & 14 & 19 \\
\hline Trial 2 & 11 & 24 \\
\hline Trial 3 & 16 & 13 \\
\hline Trial 4 & 23 & 19 \\
\hline Trial 5 & 16 & 30 \\
\hline Mean time(sec) & $\mathbf{1 5 . 6}$ & $\mathbf{2 1}$ \\
\hline
\end{tabular}

TABLE II.

Mean ACCuracy for Bulb for Two States

\begin{tabular}{|l|l|l|}
\hline \multicolumn{1}{|c|}{ Number of trials } & ON state(sec) & $\begin{array}{l}\text { OFF } \\
\text { state(sec) }\end{array}$ \\
\hline Trial 1 & $75.6 \%$ & $68.4 \%$ \\
\hline Trial 2 & $99 \%$ & $95 \%$ \\
\hline Trial 3 & $75 \%$ & $68 \%$ \\
\hline Trial 4 & $84 \%$ & $79 \%$ \\
\hline Trial 5 & $88 \%$ & $52 \%$ \\
\hline Mean Accuracy & $\mathbf{8 4 . 6 \%}$ & $\mathbf{7 2 . 4 \%}$ \\
\hline
\end{tabular}

Above Table I gives the mean response time for bulb for turn $\mathrm{ON}$ and turn OFF states. It is observed that, for five 
trials, the mean time for bulb's ON time is 15.6 seconds and bulb's OFF time is 21 seconds. OFF state takes more time than $\mathrm{ON}$ state.

Table II shows the mean accuracy for bulb for two states i.e. turn $\mathrm{ON}$ and turn OFF states for five trials. For ON state, the accuracy is $84.6 \%$ and for OFF state, the accuracy is $72.4 \%$.

\section{CONCLUSIONS}

Human brain sends signals to brain wave sensor (via Bluetooth channel). By using wave measuring unit converts raw data into suitable signals using MATLAB GUI platform. Then Commands are sent to control the devices like fan, bulb etc... By blinking the eyes there will be a change in the muscle movement, this is sensed by the Brainwave sensor and given to a MATLAB program via bluetooth, using highly Advanced RISC Machine(ARM) board and with the help of growing technology has been successfully implemented.

BCIs can have various applications over an assortment of fields, clinical and non -clinical. BCIs can give remote control in situational inability conditions, for example, space travelers and specialists, improve intellectual capacities, for example, improving consideration, official capacities, working memory, and also in non-medical applications like smart home controlling, robots controlling, security and health.

\section{REFERENCES}

[1] Anirudh Vallabhaneni et al., "Brain - Computer Interface", $1^{\text {st }}$ ed.: Springer US, 2005, pp 85-121

[2] L. Zhang, H. Leung, and K. C. C. Chan, "Information fusion based smart home control system and its application," IEEE Trans. Consumer Electron., vol. 54, no. 3, pp. 1157-1165, Aug. 2008.

[3] Ghodake, A. Brain Controlled Home Automation System. [PDF] San Diego, CA, USA: 2016 10th International Conference on Intelligent Systems and Control (ISCO), 2016, pp.1-4.

[4] S. Helal, W. Mann, H. El-Zabadani, J. King, Y. Kaddoura, and E. Jansen, "The gator tech smart house: A programmable pervasive space," IEEE Comput., vol. 38 , no. 3 , pp. 50-60, Mar. 2005.

[5] W.-H. Liau, C.-L. Wu, and L.-C. Fu, "Inhabitants tracking system in a cluttered home environment via floor load sensors," IEEE Trans. Autom. Sci. Eng., vol. 5, no. 1, pp. 1020, Jan. 2008

[6] Alrajhi, W., Alaloola, D. and Albarqawi, A. (2017). Smart Home: Toward Daily Use of BCI-Based Systems. [PDF] San Diego, CA, USA: IEEE Conference Publications, pp.1-5.

[7] Goel, K., Vohra, R. and Baths, V. (2014). Home Automation Using SSVEP \& Eye-Blink Detection Based Brain-Computer Interface. [PDF] San Diego, CA, USA: IEEE International Conference on Systems, Man, and Cybernetics, pp.4035-4036.

[8] D. H. Stefanov, Z. Bien, and W.-C. Bang, "The smart house for older persons and persons with physical disabilities: Structure, technology arrangements, and perspectives," IEEE Trans. Neural Syst. Rehabilitation Eng., vol. 12, no. 2, pp. 228-250, Jun. 2004.

[9] S. H. Kim, J. S. Kang, H. S. Park, D. Kim, and Y.-J. Kim, "UPnPZigBee internetworking architecture mirroring a multi- hop zigbee network topology," IEEE Trans. Consumer Electron., vol. 55, no. 3, pp. 1286-1294, Aug. 2009.

[10] C.-S. Li, Y.-M. Huang, and H.-C. Chao, "UPnP IPv4/IPv6 bridge for home networking environment," IEEE Trans. Consumer Electron., vol. 54, no. 4, pp. 1651-1655, Nov. 2008.

[11] T.-W. Jo, Y.-D. You, H. Choi, and H.-S. Kim, "A bluetoothUPnP bridge for the wearable computing environment," IEEE Trans. Consumer Electron., vol. 54, no. 3, pp. 1200-1205, Aug. 2008.

[12] C. Rus, K. Kontola, I. D. D. Curcio, and I. Defee, "Mobile TV content to home WLAN," IEEE Trans. Consumer Electron., vol. 54, no. 3, pp. 1038-1041, Aug. 2008.

[13] B. A. Miller, T. Nixon, C. Tai, and M. D. Wood, "Home networking with universal plug and play," IEEE Commun. Mag., vol. 39, no. 12, pp. 104-109, Dec. 2001.

[14] T. Hwang, H. Park, and E. Paik, "Location-aware UPnP AV session manager for smart home," in Proc. IEEE 1st Int. Conf. Networked Digit. Technol., Jul. 2009, pp. 106-109.

[15] B. Rebsamen, C. Guan, H. Zhang, C. Wang, C. Teo, M. H. Ang, and E. Burdet, "A brain-controlled wheelchair to navigate in familiar environments," IEEE Trans. Neural Syst. Rehabilit. Eng., vol. 18, no. 6, pp. 290-598, May 2010. 